\title{
Fixation and the stereokinetic phenomenon
}

\author{
E. S. TAUBER and L. KAUFMAN \\ Montefiore Hospital and New York University, New York, New York 10003
}

\begin{abstract}
One of two circles on a rotating disk appears to execute a planetary motion about the other circle. It is shown that the fixated circle serves as the center of rotation for the nonfixated circle. The effect of fixation is absent when polygons replace the circles. However, when one corner of an isolated square is fixated, the remaining corners rotated about the fixated corner. These planetary effects are consistent with the retinal paths followed by the elements of a display during fixation. This is not unlike the failure of position constancy associated with smooth pursuit of linearly moving targets in environments lacking a stationary visual frame of reference. In the present instance, however, the "retinal" responses occur during tracking of circularly moving targets, even in the presence of a visual frame of reference. These results are discussed in relation to the stereokinetic phenomenon. It is also shown that there is a strong interaction between the effects of fixation and the configurational features of the display. When a circle and a square are overlapping on a rotating disk, fixation of the square does not produce the perception of planetary motion. However, when the circle is fixated, the square is readily perceived as executing a planetary path about the circle. The possibility that position constancy in general is attributable to the geometry of the scene rather than to a "discounting" of information about eye movements is mentioned.
\end{abstract}

Benussi (cited in Musatti, 1924) and Musatti (1924) showed that it is possible to perceive depth when viewing flat circles and ellipsoids in rotation. An elliptical shape drawn in a disk can be perceived as a circle tilted in depth when the disk is rotated at moderate speeds. Alternatively, if the ellipse should not be seen as a circle, it exhibits a striking amoeboid motion.

Wallach, Weisz, and Adams (1956) demonstrated that such stereokinetic phenomena can be attributed to the kinetic depth effect (KDE). The contours of a shadow of a rotating three-dimensional object change in both direction and length. These changes in orientation and length of the contours of the shadow mimic what occurs when directly viewing a rotating three-dimensional object. The depth seen in the rotating shadow is known as the KDE. Illusory distortions which occur when ellipsoids are rotated in the frontal plane produce similar effects.

The illusory distortions basic to stereokinesis derive from certain properties of rotating ellipsoids (including circles). As Wallach et al. (1956) point out, a perfect circle rotating about its own center cannot be seen as rotating because all segments of the circle's contour are superimposable on each other. However, a rotating square has a determinate orientation and can therefore be seen as rotating. An

This research was partially supported by National Science Foundation Grant No. GB36976 awarded to Lloyd Kaufman. Reprint requests should be submitted to Lloyd Kaufman, Department of Psychology, New York University, 6 Washington Place, New York, New York 10003. interesting fact reported by these authors is that eccentrically rotated circles also tend to be perceived as unchanging in orientation while they are rotating. Thus, the phenomenal identity of the top of the circle is preserved despite the fact that different segments of the circle's contour occupy the uppermost position on the figure at different times.

The effects described by Wallach et al. (1956) are readily perceived by virtually all observers. In one demonstration, partially overlapping circles were viewed on a rotating disk. One circle was perceived as rotating about the other as a planet does about the sun. Moreover, since the circles tended to remain upright, they became detached from each other, with the "planet" sliding about the circumference of the "sun."

One purpose of this paper is to demonstrate that such "planetary" effects are strongly related to the fixation of the observer.

Wallach et al. (1956) also sought to explain the amoeboid distortion of rotating ellipses in terms of the tendency for circles to remain upright. Segments of the contour of an ellipse approximate the curvature of circles of different radii. If some segments of the contour of an ellipse tend to preserve their orientation in space, then other segments may appear to wobble their way around the underlying "circle." This could also simulate the conditions leading to the KDE, thereby inducing the well-known stereokinetic effect.

Squares, triangles, or other polygons have determinate orientations. The corners of such a polygon drawn on a disk can be seen as tumbling over each 
other when the disk is rotated. Hence, two overlapping triangles do not yield the sliding effect encountered with overlapping circles.

Our own observations of such stimuli suggest that eye movements play an important role in producing some of the effects. Moreover, there is an interaction between the effects of eye movements and the configurational properties of the stimuli. Conflict between the information in the display and the effects of eye movements appears to be resolved in perception in a manner that is not yet understood.

Festinger and Easton (1974) and Festinger, Sedgwick, and Holtzman (1977) have shown that, during smooth pursuit eye movements, the visual system takes very little of the position of the eyes into account in interpreting a visual scene. Thus, if the eyes should be tracking a point moving horizontally, the perceived path of a vertically moving spot will appear to be tilted. That is to say, if the horizontal eye movements are toward the right, the vertically ascending spot appears to move diagonally upward toward the left. This perceptual response is consistent with a "retinal" interpretation rather than a distal (veridical) interpretation. Therefore, the system behaves as though it is relatively ignorant of the changing position of the eyes and the resulting perception is more or less in conformity with the changing retinal configuration. Our data show that similar effects could underlie the planetary motion of circles described above.

\section{ROTATING CIRCLES}

Figure 1 is typical of some of the stimuli used by Wallach et al. (1956). As they reported, our subjects saw one of the circles glide about the other when the disk on which they were drawn was rotated at moderate speed (about $20 \mathrm{rpm}$ ). We found that the phenomenon could be observed over a wide range of speeds and viewing distances just so long as the circles could be tracked easily by the eye. In contrast with earlier findings, we noticed that clear planetary motion occurred only when subjects were able to fixate one of the circles during rotation. The fixated circle was invariably seen as the "sun," while the nonfixated circle was seen as the "planet." The same was true when three of four intertwined circles were drawn on the disk. The fixated circle was the center of rotation for the multiple planetary circles. These observations were confirmed by as many as 50 subjects, who were asked to fixate one of the circles and merely report on the paths of motion exhibited by the circles. Upon shift of fixation, there was an immediate shift in the relative perceived circular motion. The 6-in.-diam circles were drawn on a 30-in.-diam disk and viewed from distances ranging from 4 to $15 \mathrm{ft}$. All subjects reported the effect.

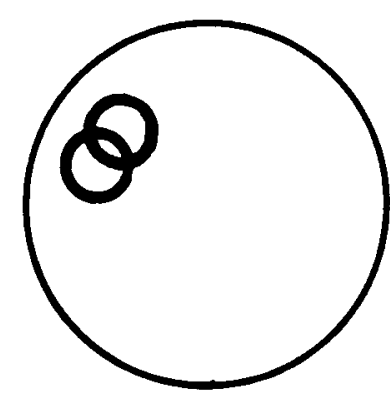

Figure 1. Two overlapping circles on a rotatable disk. Fixation of one rotating circle produces a planetary effect.

To provide a better test of the observation that the relative motions of the circles were determined by fixation, we utilized the Haidinger's brush technique employed by Kaufman and Richards (1969) in a study of spontaneous fixation tendencies. In the present instance, however, the brush was permanently visible to each of our five subjects, because the polarizer was rotating continuously rather than intermittently. The subject was seated before a rotating linear polarizing filter. He viewed a 30 -in.diam disk through the filter from a distance of about $7 \mathrm{ft}$. The disk was illuminated by a projector with a Corning 4-60 glass filter placed before its lens so that blue light was falling on the disk. Otherwise the room was darkened. Circles similar to those shown in Figure 1 were drawn in India ink on the white disk. The circles were 6 in. in diameter, thereby subtending visual angles of $4.08 \mathrm{deg}$ of arc. The subject was instructed to keep the "propeller"' (brush) inside either the leading or trailing circle as the disk was rotated at $20 \mathrm{rpm}$ clockwise. All five subjects reported that the circle containing the propeller was the "sun" while the other circle was the "planet." When the brush was maintained on the center of the disk rather than in one of the circles, the planetary motion was absent. Instead, one of the circles was seen as trailing the other, although both circles remained "upright" as reported by Wallach et al. Since identical results were obtained simply by instructing the subject to fixate one or the other circle or the center of the disk, we discontinued using the brush technique in the remaining experiments.

\section{ROTATING SQUARES}

When squares subtending $4 \mathrm{deg}$ of arc on each side (Figure 2) were presented on the disk to 10 subjects, planetary motion could not be seen regardless of where fixation was maintained.

It should be noted that there is a retinal planetary motion of all points on a rotating disk relative to the fixated point. If the line of sight is coincident with a particular physical point on a disk, then all other 


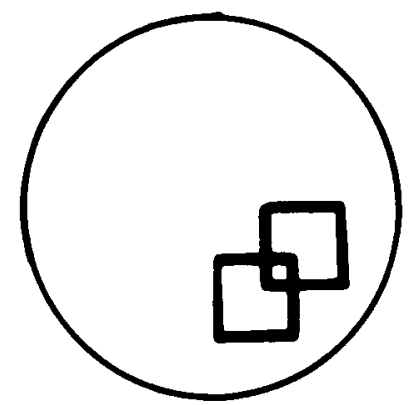

Figure 2. Two overlapping squares on rotatable disk. Planetary effect does not occur.

points on the rotating disk pass through an arc of 360 deg about the fixation point for each revolution of the disk. With this geometrical fact in mind, we also separated two smaller squares so that they occupied different places on the disk, as in Figure 3. The 1-in. squares were separated by a center-tocenter distance of 6 in. and viewed by each subject at a distance of 7 and $15 \mathrm{ft}$. With fixation of one of these squares, the other square does in fact execute planetary motion about the fixated square in the retinal image. Here, too, none of our 10 subjects were able to see clear planetary motion. When the squares of Figure 3 (which is not drawn to scale) were replaced with small circles with diameters equal to the diagonals of the squares, the same subjects had an immediate experience of planetary motion with fixation of one of the circles.

One additional observation was made by eight new subjects who viewed the rotating squares of the previous experiment from a distance of $3 \mathrm{ft}$. The fixated square was invariably seen as spinning about the same point within its boundaries, while the nonfixated square did not appear to spin at as fast a rate. When asked, the eight subjects reported the fixated square to be rotating about itself many times faster than the nonfixated square. The same was true of triangles. With careful fixation, it was possible for the authors to see that a fixated corner of a square can serve as the center of rotation for the remaining three nonfixated corners. This was also true of larger squares, e.g., 4 in. on a side, viewed from many different distances. This in conformity with the actual changes taking place on the retina. Nevertheless, squares do not appear to rotate about each other when they replace circles on the rotating disk. The foregoing phenomena were so compelling that many casual observers spontaneously reported them.

\section{SQUARE AND CIRCLE}

A square (4 in. on each side) and a circle (5.7-in. diameter) were overlapped on a disk, as shown in Figure 4 , and viewed by five new subjects and the authors from a distance of $7 \mathrm{ft}$.
When the circle was fixated, the square appeared to be sliding around the circle. However, when the square was fixated, this planetary motion could not be seen. The circle retained its position in space relative to the square. These phenomena were also readily reported by many casual observers who viewed the rotating disk from many different distances. It is clear that the configurational properties of such a display inhibits the planetary motion, since the circle is always seen as overlapping one corner of the square. However, since the circle tends to remain upright and does not possess orientational information, it is possible to see the square spin about the circle.

\section{ELLIPSES}

As already indicated, an ellipse is not strongly directional despite its having axes of different length. When viewed on a rotating disk, there is a choice, perceptually speaking, between a deforming planar image and a tumbling rigid three-dimensional image (a circle in depth). We have repeated and confirmed these earlier findings. The lack of directionality of an ellipse is supported by the fact that overlapping ellipses behave in much the same manner as do overlapping circles.

In addition to this replication, we placed an ellipse with its major axis along a diameter of an enclosing circle which was itself drawn eccentrically relative to

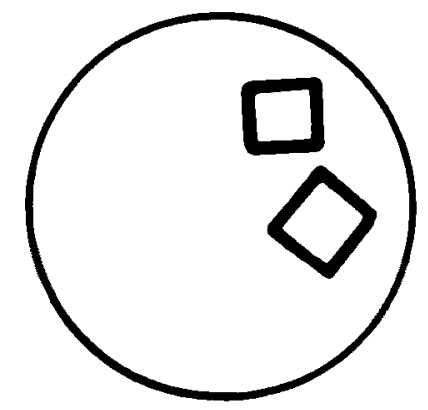

Figure 3. There is no planetary effect even when small squares are separated on a disk.

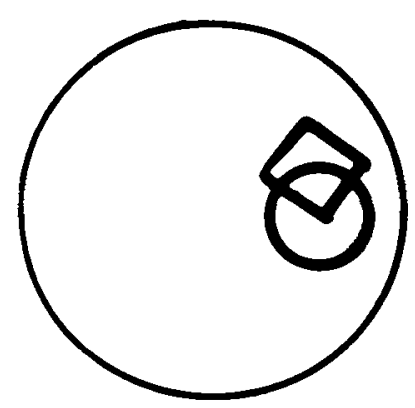

Figure 4. Fixation of circle produces planetary effect while the effect is absent with fixation of square. 
the rotating disk. This ellipse tended to be seen as a circle in depth when rotated. However, when the major axis of the ellipse was placed on a chord of the enclosing circle, so that its contour was tangential to the inner boundary of the circle, the curious amoeboid motion reported by Wallach et al. was the predominant experience. It is likely that some interaction of the adjacent contours of the circle and the ellipse can affect the perception of the rotating ellipse. The foregoing observations were made by the authors and casual observers and were so easily seen that it was considered to be unnecessary to employ additional formal observations.

\section{DISCUSSION}

As demonstrated by the work of Festinger and his colleagues (Festinger \& Easton, 1974; Festinger et al., 1977), when a slowly moving point is fixated so that the eye executes a slow pursuit movement, the resulting perception of the paths of other moving points more accurately described in terms of the paths of the points actually present on the retina rather than the paths of the points in the environment. These results were obtained under conditions in which the points were seen in a dark field and in the absence of a visible stationary framework. We have now shown that similar results may be obtained in the presence of a visible framework provided that the motion of the stimuli is circular. It is therefore suggested that when moving luminous points are tracked by the eyes within a visible framework, effects analogous to those found by Festinger and his colleagues would be present provided that the paths of motion were circular. Also, the implied loss to the perceptual system during slow eye movements of information about the movement of the eyes can account for the distortions perceptible in the stimuli that produce the stereokinetic effect. It is the "parallax" imitated by the perception of the relative retinal paths that probably underlies the stereokinetic effect itself. It is also established that when configurational information is provided so that the orientations of stimuli relative to each other remain constant even during rotary motion, as when the square is fixated in the overlapping circle and square, the perceptual system resolves this conflicting situation in favor of the configurational cues. In a very real sense, it appears that the perceptual system is providing a cognitive solution to the conflict. This interpretation is consistent with Rock's (1975) view that the perceptual system operates to provide the most rational solution to conflicting cues. We suggest that position constancy in general, i.e., the fact that objects apparently preserve their positions in the visual world despite the shifting of the pattern of retinal stimulation during eye and head movements, is based upon invariant configurational information rather than a "discounting" of the eye and head movements.

\section{REFERENCES}

Festinger, L., \& EASton, A. M. Inferences about the efferent system based on a perceptual illusion produced by eye movements. Psychological Review, 1974, 81, 44-58.

Festinger, L., Sedgwick, H. A., \& Holtzman, J. D. Visual perception during smooth pursuit eye movements. Psychological Review, 1977, in press.

Kaufman, L., \& Richards, W. Spontaneous fixation tendencies for visual forms. Perception \& Psychophysics, 1969, 5, 85-88.

Mussati, C. L. Sui fenomeni stereokinetici. Archivio Italiano di Psicologia, 1924, 3, 105-120.

Rock, I. An introduction to perception. New York: Macmillan, 1975.

Wallach, H., Weisz, W., \& Adams, P. A. Circles and derived figures in rotation. American Journal of Psychology, 1956, 69, 48-59.

(Received for publication December 27, 1976; revision accepted June $1,1977$. 